\title{
On the Flexibility of Topp Leone Exponentiated Inverse Exponential Distribution
}

\author{
Sule Ibrahim ${ }^{1}$, Sani Ibrahim Doguwa ${ }^{1}$, Audu Isah ${ }^{2}$, Haruna Muhammad Jibril $^{3}$ \\ ${ }^{1}$ Department of Statistics, Faculty of Physical Sciences, Ahmadu Bello University, Zaria, Nigeria \\ ${ }^{2}$ Department of Statistics, School of Physical Sciences, Federal University of Technology, Minna, Nigeria \\ ${ }^{3}$ Department of Mathematics, Faculty of Physical Sciences, Ahmadu Bello University, Zaria, Nigeria \\ Email address: \\ ibrahimsule76@yahoo.com (S. Ibrahim), sidoguwa@gmail.com (S. I. Doguwa), aisah@futminna.edu.ng (A. Isah), \\ alharun2004@yahoo.com (H. M. Jibril)

\section{To cite this article:} \\ Sule Ibrahim, Sani Ibrahim Doguwa, Audu Isah, Haruna Muhammad Jibril. On the Flexibility of Topp Leone Exponentiated Inverse \\ Exponential Distribution. International Journal of Data Science and Analysis. Special Issue: Data Science. Vol. 6, No. 3, 2020 , pp. 83-89. \\ doi: $10.11648 /$ j.ijdsa.20200603.12
}

Received: May 1, 2020; Accepted: June 10, 2020; Published: July 17, 2020

\begin{abstract}
In this paper, we introduced a new continuous probability distribution called the Topp Leone exponentiated inverse exponential distribution with three parameters. We studied the nature of proposed distribution with the help of its mathematical and statistical properties such as quantile function, ordinary moments, moment generating function, survival function and hazard function. The probability density function of order statistic for this distribution was also obtained. We performed classical estimation of parameters by using the technique of maximum likelihood estimate. The proposed model was applied to two reallife datasets. The first data set has to do with patients with cancer of tongue with aneuploidy DNA profile and the second data set has to do with patients who were diagnosed with hypertension and received at least one treatment related to hypertension. The results showed that the new distribution provided better fit than other distributions presented. As such, it can be categorically said that the Topp Leone exponentiated inverse exponential distribution is good distribution in modeling survival data.
\end{abstract}

Keywords: Distribution, Inverse Exponential, Ordinary Moment, Parameter, Quantile Function

\section{Introduction}

The amount of data available for analysis is growing increasingly faster, requiring new probabilistic distributions to better describe each phenomenon. Computer based tools allow the use of more complex distributions with a larger number of parameters to better study sizeable volume of data.

The two parameter generalized exponential distribution is a particular member of the three parameter exponentiated Weibull distribution, introduced by Mudholkar and Srivastava [1]. Moreover, the exponentiated Weibull distribution is a special case of general class of exponentiated distributions proposed by Gupta et al., [2] as $F(t)=[\mathrm{G}(\mathrm{t})]^{\alpha}$, where $\mathrm{G}(\mathrm{t})$ is the baseline distribution function. It is observed by Gupta and Kundu [3], that the two parameter generalized exponential distribution can be used quite effectively to analyze positive life time data, particularly, in place of the two parameter gamma or two parameter Weibull distributions. Moreover, when the shape parameter, $\alpha=1$, it coincides with the one parameter exponential distribution. Therefore, all the three distributions, namely generalized exponential, Weibull and gamma are all extensions of generalization of the one parameter exponential distribution in different ways. Some recent generalizations of the exponential distribution are; Exponentiated Weibull-Lomax by Hassan and Abd-Allah [4], Exponentiated Inverse Power Lindley by Jan et al. [5], Exponentiated Lomax Geometric by Hassan and Abdelghafar [6], Exponentiated Generalized Weibull Gompertz by El-Bassiouny et al., [7].

The Inverse Exponential (IEx) distribution was introduced by Keller and Kamath [8] and since then, it has been studied and discussed as a life time model. The IEx distribution is capable of modeling data sets with inverted bathtub failure rate. It is a modification of the exponential distribution and has an advantage of not having a constant failure rate.

Several generalizations of IEx distribution have been studied. An approach to the construction of flexible parametric models is to embed appropriate competing models into a larger model by adding one or more shape parameter (s). Some recent generalizations of IEx distribution are: The 
transmuted inverse exponential distribution by Oguntunde and Adejumo [9], Kumaraswamy inverse exponential distribution by Oguntunde et al., [10], transmuted inverse exponential distribution Oguntunde et al., [11].

Ibrahim et al., [12] proposed a new family of continuous distributions called the Topp Leone Exponentiated-G (TLExG) family with two extra positive shape parameters $\alpha$ and $\theta$. For an arbitrary baseline cumulative distribution function (cdf) $H(x, \varphi)$, the TLEx-G family with two extra positive shape parameters $\alpha$ and $\theta$ has cdf and probability density function $(\mathrm{pdf})$ for $(\mathrm{x}>0)$ given by

$$
F(x ; \alpha, \theta, \varphi)=\left\{1-\left[1-H(x, \varphi)^{\alpha}\right]^{2}\right\}^{\theta}
$$

and

$$
f(x ; \alpha, \theta, \varphi)=2 \alpha \theta h(x ; \varphi) H(x ; \varphi)^{\alpha-1}\left[1-H(x ; \varphi)^{\alpha}\right]\left\{1-\left[1-H(x ; \varphi)^{\alpha}\right]^{2}\right\}^{\theta-1}
$$

$$
x>0, \alpha, \theta, \varphi>0
$$

respectively.

Whereh $(x ; \varphi)=\frac{d H(x ; \varphi)}{d x}$ is the baseline pdf, $\alpha$ and $\theta$ are positive shape parameters.

The cdf and pdf of the IEx distribution are given as

$$
H(x ; \beta)=e^{-\left(\frac{\beta}{x}\right)}
$$

and

$$
\begin{gathered}
h(x ; \beta)=\left(\frac{\beta}{x^{2}}\right) e^{-\left(\frac{\beta}{x}\right)} \\
x>0, \beta>0
\end{gathered}
$$

The main objectives of this paper are to obtain a more flexible model by inducting two extra shape parameters to the IEx distribution and to improve goodness-of-fit of the distribution to model real-life data.

Based on Ibrahim et al., [12], we derive the Topp Leone exponentiated inverse exponential (TLExIEx) distribution and provide a comprehensive description of some of its mathematical and statistical properties. The paper is outlined as follows. In Section 2, the Topp Leone exponentiated inverse exponential distribution is defined and also the graphs of its pdf and hazard rate function are presented. In Section 3, some mathematical and statistical properties including the moments, quantile function (qf), moment generating function (mgf) and survival and hazard functions are derived. The pdf of the order statistic is derived in section 4. In Section 5, the maximum likelihood estimates (MLEs) of the model parameters is obtained. In Section 6, application of the model to two real-life datasets is presented and its fitness and flexibility with other competing models is assessed. Finally, in section 7, some concluding remarks are provided.

\section{The Topp Leone Exponentiated Inverse Exponential (TLExIEx) Distribution}

In this section, we define a new model called TLExIEx model and provide some plots of its pdf and hazard rate function (hrf). The TLExIEx cdf is obtained by inserting (3) into (1) given as:

$$
F(x ; \alpha, \theta, \beta)=\left\{1-\left[1-\left(e^{-\left(\frac{\beta}{x}\right)}\right)^{\alpha}\right]^{2}\right\}^{\theta}
$$

and

$$
f(x ; \alpha, \theta, \beta)=2 \alpha \theta\left(\frac{\beta}{x^{2}}\right) e^{-\left(\frac{\beta}{x}\right)}\left[e^{-\left(\frac{\beta}{x}\right)}\right]^{\alpha-1}\left[1-\left(e^{-\left(\frac{\beta}{x}\right)}\right)^{\alpha}\right]\left\{1-\left[1-\left(e^{-\left(\frac{\beta}{x}\right)}\right)^{\alpha}\right]^{2}\right\}^{\theta-1}
$$

where,

$\frac{d F(x ; \alpha, \theta, \beta)}{d x}=f(x ; \alpha, \theta, \beta)$ is the pdf of the TLExIEx distribution

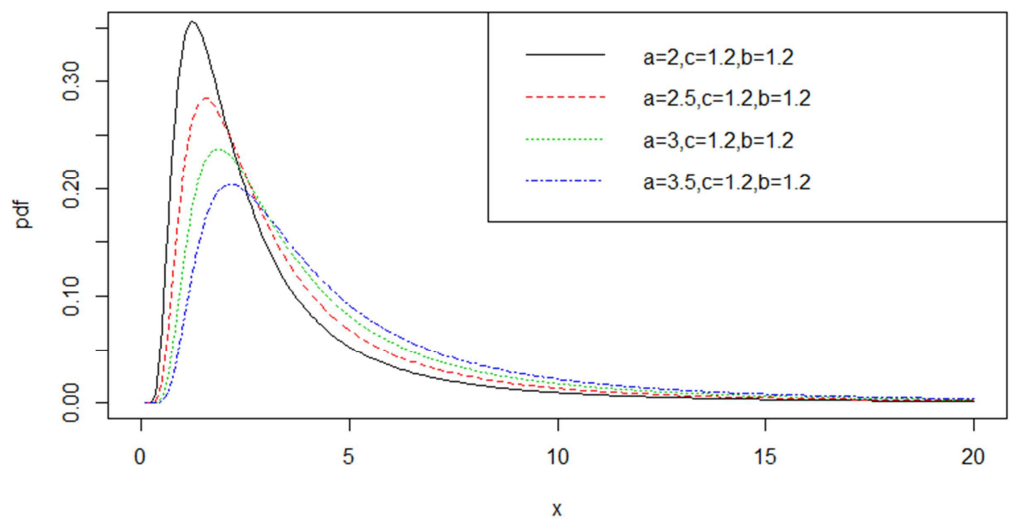

Figure 1. The pdf plot of TLExIEx distribution with different parameter values $(a=\alpha, b=\beta, c=\theta)$. 


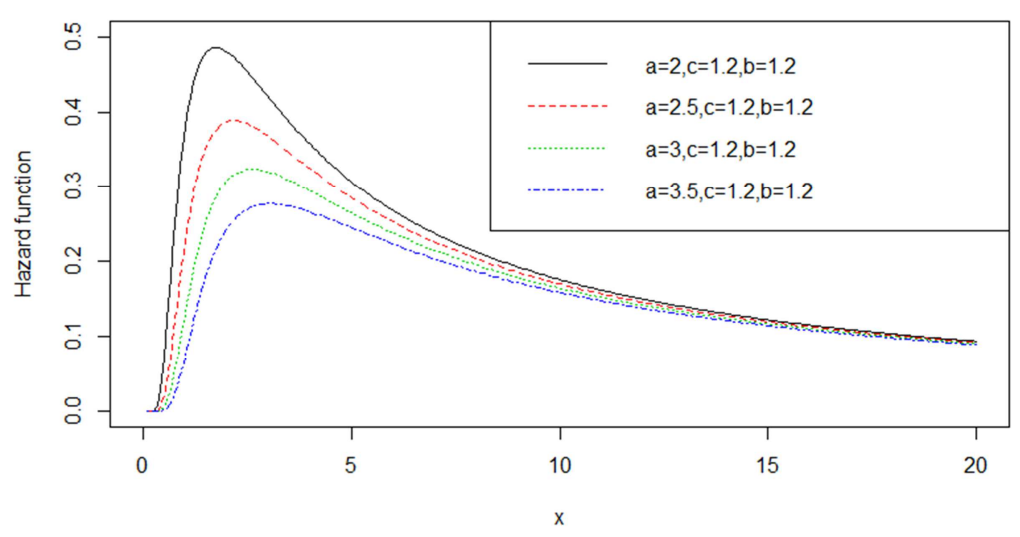

Figure 2. The hrf plot of TLExIEx distribution with different parameter values $(a=\alpha, b=\beta, c=\theta)$.

The additional shape parameters $\alpha$ and $\theta$ are sought as a manner to furnish a more flexible TLExIEx distribution.

\section{Mathematical and Statistical Properties}

In this section, the quantile function, moments, moment generating function, reliability measure will be derived.

\subsection{Quantile Function}

The quantile function is a useful measure for describing the distribution of a random variable. It plays a key role when simulating random numbers and can also be used to compute the median, kurtosis and skewness of the distribution of a random variable.

If $u$ has a uniform $U(0,1)$ distribution, the quantile function can be obtained by inverting (5) as:

$$
\begin{aligned}
& u=\left\{1-\left[1-\left[e^{-\left(\frac{\beta}{x}\right)}\right]^{\alpha}\right]^{2}\right\}^{\theta} \\
& u^{\frac{1}{\theta}}=1-\left[1-\left[e^{-\left(\frac{\beta}{x}\right)}\right]^{\alpha}\right]^{2} \\
& 1-u^{\frac{1}{\theta}}=\left[1-\left[e^{-\left(\frac{\beta}{x}\right)}\right]^{\alpha}\right]^{2} \\
& E\left(X^{r}\right)=\int_{0}^{\infty} x^{r} 2 \alpha \theta\left(\frac{\beta}{x^{2}}\right) e^{-\left(\frac{\beta}{x}\right)}\left[e^{-\left(\frac{\beta}{x}\right)}\right]^{\alpha-1}\left[1-\left(e^{-\left(\frac{\beta}{x}\right)}\right)^{\alpha}\right]\left\{1-\left[1-\left(e^{-\left(\frac{\beta}{x}\right)}\right)^{\alpha}\right]^{2}\right\}^{\theta-1} d x \\
& E\left(X^{r}\right)=\int_{0}^{\infty} x^{r} 2 \alpha \theta\left(\frac{\beta}{x^{2}}\right)\left[e^{-\left(\frac{\beta}{x}\right)}\right]^{\alpha}\left[1-\left(e^{-\left(\frac{\beta}{x}\right)}\right)^{\alpha}\right]\left\{1-\left[1-\left(e^{-\left(\frac{\beta}{x}\right)}\right)^{\alpha}\right]^{2}\right\}^{\theta-1} d x \\
& E\left(X^{r}\right)=2 \alpha \theta \beta \int_{0}^{\infty} x^{r-2}\left[e^{-\left(\frac{\beta}{x}\right)}\right]^{\alpha}\left[1-\left(e^{-\left(\frac{\beta}{x}\right)}\right)^{\alpha}\right]\left\{1-\left[1-\left(e^{-\left(\frac{\beta}{x}\right)}\right)^{\alpha}\right]^{2}\right\}^{\theta-1} d x
\end{aligned}
$$

Using binomial expansion with the relation

$$
(1-x)^{\theta-1}=\sum_{i=0}^{\infty} \frac{(-1)^{i} \Gamma(\theta)}{i ! \Gamma(\theta-i)}
$$

and 


$$
(1-x)^{\theta}=\sum_{i=0}^{\infty} \frac{(-1)^{i} \Gamma(\theta+1)}{i ! \Gamma(\theta+1-i)}
$$

Using the relation (10) on the last term in (9), we have

$$
\left\{1-\left[1-\left(e^{-\left(\frac{\beta}{x}\right)}\right)^{\alpha}\right]^{2}\right\}^{\theta-1}=\sum_{i=0}^{\infty} \frac{(-1)^{i} \Gamma(\theta)}{i ! \Gamma(\theta-i)}\left[1-\left(e^{-\left(\frac{\beta}{x}\right)}\right)^{\alpha}\right]^{2 i}
$$

Substituting into (9), we have

$$
\begin{gathered}
E\left(X^{r}\right)=2 \alpha \theta \beta \sum_{i=0}^{\infty} \frac{(-1)^{i} \Gamma(\theta)}{i ! \Gamma(\theta-i)} \\
\int_{0}^{\infty} x^{r-2}\left[e^{-\left(\frac{\beta}{x}\right)}\right]^{\alpha}\left[1-\left(e^{\left.-\left(\frac{\beta}{x}\right)\right)^{\alpha}}\right]^{2 i+1} d x\right. \\
{\left[1-\left(e^{-\left(\frac{\beta}{x}\right)}\right)^{\alpha}\right]^{2 i+1}=\sum_{j=0}^{\infty} \frac{(-1)^{j} \Gamma(2(i+1))}{j ! \Gamma(2(i+1)-j)}\left[e^{\left.-\left(\frac{\beta}{x}\right)\right]^{\alpha j}}\right.} \\
E\left(X^{r}\right)=2 \alpha \theta \beta \sum_{i=0}^{\infty} \frac{(-1)^{i} \Gamma(\theta)}{i ! \Gamma(\theta-i)} \sum_{j=0}^{\infty} \frac{(-1)^{j} \Gamma(2(i+1))}{j ! \Gamma(2(i+1)-j)} \int_{0}^{\infty} x^{r-2}\left[e^{-\frac{\alpha \beta}{x}}\right]^{(j+1)} d x .
\end{gathered}
$$

From (12),

Let $y=\frac{\alpha \beta}{x}(j+1), x=\frac{\alpha \beta}{y}(j+1), d x=\frac{-d y}{(j+1) \alpha \beta x^{2}}$

Then,

$$
\begin{gathered}
E\left(X^{r}\right)=2 \alpha \theta \beta \sum_{i=0}^{\infty} \frac{(-1)^{i} \Gamma(\theta)}{i ! \Gamma(\theta-i)} \sum_{j=0}^{\infty} \frac{(-1)^{j} \Gamma(2(i+1))}{j ! \Gamma(2(i+1)-j)} \\
\int_{0}^{\infty}\left(\frac{\alpha \beta}{y}(j+1)\right)^{r-2} e^{-\left(\frac{\alpha \beta(j+1)}{\alpha \beta(j+1) y}\right)} \frac{d y}{\alpha \beta(j+1)} \\
E\left(X^{r}\right)=2 \alpha \theta \beta \sum_{i=0}^{\infty} \sum_{j=0}^{\infty}(-1)^{i+j} \frac{\Gamma(\theta) \Gamma(2(i+1))}{i ! j ! \Gamma(\theta-i) \Gamma(2(i+1)-j)}(\alpha \beta(j+1))^{r-1} \int_{0}^{\infty} y^{-r} e^{-y} d y \\
E\left(X^{r}\right)=2 \alpha^{r} \theta \beta^{r} \sum_{i=0}^{\infty} \sum_{j=0}^{\infty}(-1)^{i+j} \frac{\Gamma(\theta) \Gamma(2(i+1))}{i ! j ! \Gamma(\theta-i) \Gamma(2(i+1)-j)}(j+1)^{r-1} \Gamma(1-r)
\end{gathered}
$$

Equation (13) is the $r^{\text {th }}$ moment of the TLExIEx distribution. The mean of the distribution will be obtained by setting $r=1$ in (13).

\subsection{Moment Generating Function (MGF)}

The mgf of $X$ can be obtained using the equation

$$
\begin{gathered}
M_{x}(t)=E\left(e^{t x}\right)=\int_{-\infty}^{\infty} e^{t x} f(x) d x \\
E\left(e^{t x}\right)=2 \alpha \theta \beta \int_{0}^{\infty} x^{-2} e^{t x}\left[e^{-\left(\frac{\beta}{x}\right)}\right]^{\alpha}\left[1-\left(e^{-\left(\frac{\beta}{x}\right)}\right)^{\alpha}\right]\left\{1-\left[1-\left(e^{-\left(\frac{\beta}{x}\right)}\right)^{\alpha}\right]^{2}\right\}^{\theta-1} d x
\end{gathered}
$$

Following the process of moments above, we have

$$
E\left(e^{t x}\right)=2 \alpha \theta \beta \sum_{i=0}^{\infty} \sum_{j=0}^{\infty}(-1)^{i+j} \frac{\Gamma(\theta) \Gamma(2(i+1))}{i ! j ! \Gamma(\theta-i) \Gamma(2(i+1)-j)} \int_{0}^{\infty} x^{-2} e^{t x}\left[e^{-\frac{\alpha \beta}{x}}\right]^{(j+1)} d x
$$

Let $y=\frac{\alpha \beta}{x}(j+1), x=\frac{\alpha \beta}{y}(j+1), d x=\frac{-d y}{(j+1) \alpha \beta x^{2}}$

Using binomial expansion,

$$
e^{t x}=\sum_{m=0}^{\infty} \frac{t^{m} x^{m}}{m !}
$$




$$
\begin{gathered}
E\left(e^{t x}\right)=2 \alpha \theta \beta \sum_{i=0}^{\infty} \sum_{j=0}^{\infty} \sum_{m=0}^{\infty}(-1)^{i+j} \frac{\Gamma(\theta) \Gamma(2(i+1))}{i ! j ! \Gamma(\theta-i) \Gamma(2(i+1)-j)} \frac{t^{m}}{m !} \int_{0}^{\infty} x^{m-2} e^{-\left(\frac{\alpha \beta(j+1)}{\alpha \beta(j+1) y}\right)} d x E\left(e^{t x}\right) \\
=2 \alpha \theta \beta \sum_{i=0}^{\infty} \sum_{j=0}^{\infty} \sum_{m=0}^{\infty}(-1)^{i+j} \frac{\Gamma(\theta) \Gamma(2(i+1))}{i ! j ! \Gamma(\theta-i) \Gamma(2(i+1)-j)} \frac{t^{m}}{m !} \int_{0}^{\infty}\left(\frac{\alpha \beta}{y}(j+1)\right)^{m-2} e^{-\left(\frac{\alpha \beta(j+1)}{\alpha \beta(j+1) y}\right)} \frac{d y}{\alpha \beta(j+1)} \\
E\left(e^{t x}\right)=2 \alpha \theta \beta \sum_{i=0}^{\infty} \sum_{j=0}^{\infty} \sum_{m=0}^{\infty}(-1)^{i+j} \frac{\Gamma(\theta) \Gamma(2(i+1))}{i ! j ! \Gamma(\theta-i) \Gamma(2(i+1)-j)} \frac{t^{m}}{m !}(\alpha \beta(j+1))^{m-1} \int_{0}^{\infty} y^{-m} e^{-y} d y \\
E\left(e^{t x}\right)=2 \theta \sum_{i=0}^{\infty} \sum_{j=0}^{\infty} \sum_{m=0}^{\infty}(-1)^{i+j} \frac{\Gamma(\theta) \Gamma(2(i+1)) t^{m} \alpha^{m} \beta^{m}(j+1)^{m-1}}{i ! j ! m ! \Gamma(\theta-i) \Gamma(2(i+1)-j)} \Gamma(1-\mathrm{m})
\end{gathered}
$$

\subsection{ReliabilityFunction}

The reliability function is also known as survival function, which is the probability of an item not failing prior to some time. It can be defined as

$$
\begin{gathered}
R(x ; \alpha, \theta, \beta)=1-F(x ; \alpha, \theta, \beta) \\
R(x ; \alpha, \theta, \beta)=1-\left\{1-\left[1-\left(e^{-\left(\frac{\beta}{x}\right)}\right)^{\alpha}\right]^{2}\right\}^{\theta}
\end{gathered}
$$

\subsection{Hazard Function}

The hazard rate function is an important measure use to characterize a life phenomenon. It is given as

$$
\begin{gathered}
\tau(x ; \alpha, \theta, \beta)=\frac{f(x ; \alpha, \theta, \beta)}{R(x ; \alpha, \theta, \beta)} \\
\tau(x ; \alpha, \theta, \beta)=\frac{2 \alpha \theta\left(\frac{\beta}{x^{2}}\right)\left[e^{-\left(\frac{\beta}{x}\right)}\right]^{\alpha}\left[1-\left(e^{-\left(\frac{\beta}{x}\right)}\right)^{\alpha}\right]\left\{1-\left[1-\left(e^{-\left(\frac{\beta}{x}\right)}\right)^{\alpha}\right]^{2}\right\}^{\theta-1}}{1-\left\{1-\left[1-\left(e^{-\left(\frac{\beta}{x}\right)}\right)^{\alpha}\right]^{2}\right\}^{\theta}}
\end{gathered}
$$

\section{Order Statistic}

Order statistic makes its appearance in many areas of statistical theory and practice. Let $X_{(1)}, X_{(2)}, \ldots, X_{(n)}$ be $n$ independent random variable from the TLExIEx distribution and let $X_{(1)} \leq X_{(2)} \leq, \ldots, \leq X_{(n)}$ be their corresponding order statistic.

Let $F_{r: n}(x)$ and $f_{r: n}(x), r=1,2,3, \ldots, n$ denote the cdf and pdf of the $r^{\text {th }}$ order statistic $X_{r: n}$ respectively. The pdf of $X_{r: n}$ is given as

$$
\begin{gathered}
f_{r: n}(x)=\frac{1}{B(r, n-r+1)} f(x)[F(x)]^{r-1}[1-F(x)]^{n-r} \\
f_{r: n}(x)=\frac{1}{B(r, n-r+1)} \sum_{i=0}^{n-r}(-1)^{i} f(x)[F(x)]^{r+i-1} \\
f_{r: n}(x)=\frac{1}{B(r, n-r+1)} \sum_{i=0}^{n-r}(-1)^{i} 2 \alpha \theta\left(\frac{\beta}{x^{2}}\right)\left[e^{\left.-\left(\frac{\beta}{x}\right)\right]^{\alpha}}\right. \\
X\left[1-\left(e^{-\left(\frac{\beta}{x}\right)}\right)^{\alpha}\right]\left\{1-\left[1-\left(e^{-\left(\frac{\beta}{x}\right)}\right)^{\alpha}\right]^{2}\right\}^{\theta-1}\left\{1-\left[1-\left(e^{-\left(\frac{\beta}{x}\right)}\right)^{\alpha}\right]^{2}\right\}^{\theta(r+i-1)} \\
f_{r: n}(x)=\frac{1}{B(r, n-r+1)} 2 \alpha \theta \beta \sum_{i=0}^{n-r} \sum_{j=0}^{\infty} \sum_{k=0}^{\infty}(-1)^{i+j+k}\left(\begin{array}{c}
\theta(r+i)-1 \\
j
\end{array}\right)\left(\begin{array}{c}
2 j+1 \\
k
\end{array}\right) \frac{1}{x^{2}}\left[\left(e^{-\left(\frac{\beta}{x}\right)}\right)^{\alpha}\right]^{k+1}
\end{gathered}
$$

Equation (23) is the pdf of the $r^{\text {th }}$ order statistic of the TLExIEx distribution from which we can obtain the 
minimum order statistic by setting $r=1$ and maximum order statistic by setting $r=n$.

\section{Estimation}

In this section, we consider the estimation of the unknown parameters of the TLExIEx distribution by maximum likelihood estimation method for the complete samples. The maximum likelihood estimates (MLEs) enjoy desirable properties that can be used when constructing confidence intervals and deliver simple approximations that work well in finite samples.

Let $x_{1}, x_{2}, \ldots, x_{n}$ be independent and identically distributed observed random sample of size $n$ from the TLExIEx distribution. Then, the log-likelihood function based on observed sample for the vector of parameter $\varnothing=(\alpha, \theta, \beta)^{T}$ is given by

$$
\begin{gathered}
L(\varnothing)=n \log (2)+n \log (\alpha)+n \log (\theta)+n \log (\beta)+\sum_{i=1}^{n} \log \left(\frac{1}{x_{i}^{2}}\right)-\sum_{i=1}^{n}\left(\frac{\beta}{x_{i}}\right)^{\alpha}+\sum_{i=1}^{n} \log \left(\left(e^{-\left(\frac{\beta}{x_{i}}\right)}\right)^{\alpha}\right)+(\theta- \\
\text { 1) } \sum_{i=1}^{n} \log \left(1-\left(1-\left(e^{-\left(\frac{\beta}{x_{i}}\right)}\right)^{\alpha}\right)^{2}\right)
\end{gathered}
$$

The components of the score vector, say $\Delta L(\varnothing)=\left(\frac{\partial L(\varnothing)}{\partial \alpha}, \frac{\partial L(\varnothing)}{\partial \beta}, \frac{\partial L(\varnothing)}{\partial \theta}\right)$ are

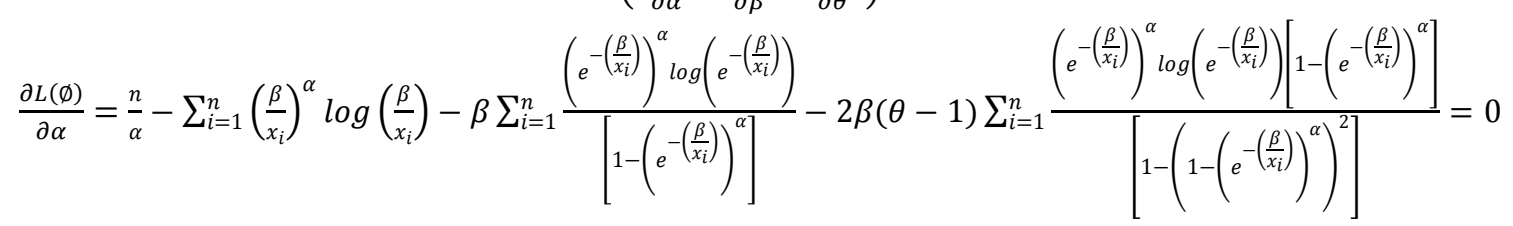

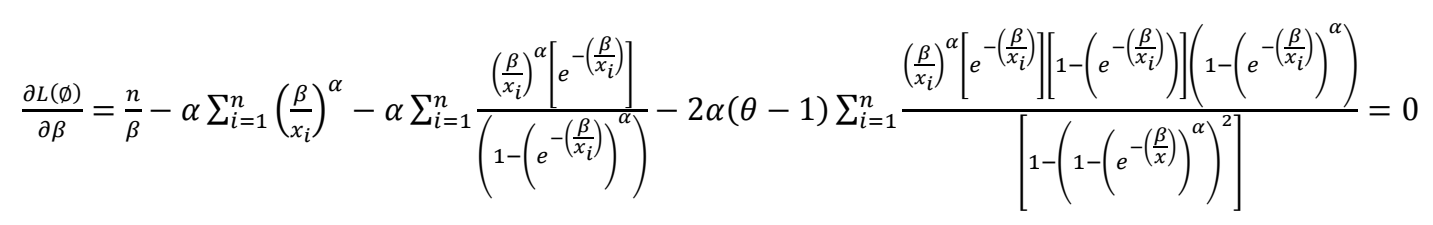

$$
\begin{aligned}
& \frac{\partial L(\varnothing)}{\partial \theta}=\frac{n}{\theta}+\sum_{i=1}^{n} \log \left[1-\left(1-\left(e^{-\left(\frac{\beta}{x_{i}}\right)}\right)^{\alpha}\right)^{2}\right]=0
\end{aligned}
$$

The maximum likelihood estimates (MLEs) of the parameters are the solutions of the non-linear equations $\Delta L(\varnothing)=0$, which are solved iteratively using a package in $R$ called AdequacyModel.

\section{Application to Real-life Data}

In this section, the proposed TLExIEx distribution is applied to two real-life data sets. We observe its flexibility over some existing distributions. The results for the analysis in this present study re obtained using R-software. Meanwhile, the distribution with the lowest AIC, CAIC, BIC is considered the best. The pdf of the distributions taken are as follow:

1. Topp Leone Exponentiated Inverse Exponential distribution.

$$
\begin{aligned}
& f(x ; \alpha, \theta, \beta)=2 \alpha \theta\left(\frac{\beta}{x^{2}}\right) e^{-\left(\frac{\beta}{x}\right)}\left[e^{-\left(\frac{\beta}{x}\right)}\right]^{\alpha-1} \\
& {\left[1-\left(e^{-\left(\frac{\beta}{x}\right)}\right)^{\alpha}\right]\left\{1-\left[1-\left(e^{-\left(\frac{\beta}{x}\right)}\right)^{\alpha}\right]^{2}\right\}^{\theta-1}}
\end{aligned}
$$

2. Topp Leone Inverse Exponential distribution.

$$
f(x ; \beta, \theta)=2 \theta\left(\frac{\beta}{x^{2}}\right) e^{-\left(\frac{\beta}{x}\right)}
$$

3. Exponentiated Inverse Exponential distribution.

$$
f(x ; \beta, \alpha)=\frac{\alpha \beta}{x^{2}} e^{-\left(\frac{\beta}{x}\right)}\left(e^{-\left(\frac{\beta}{x}\right)}\right)^{\alpha-1}
$$

4. Inverse Exponential distribution.

$$
f(x ; \beta)=\left(\frac{\beta}{x^{2}}\right) e^{-\left(\frac{\beta}{x}\right)}
$$

5. Exponential distribution.

$$
f(x ; \beta)=\beta e^{-\beta x}
$$

Data set 1:

The data set in Table 1 represents the death times (in weeks) of patients with cancer of tongue with aneuploidy DNA profile. The data set has been previously used by Klein and Moeschberger [13].

Table 1. The table showing the data set of death times of patients with cancer of the tongue.

$1,3,3,4,10,13,13,16,16,24,26,27,28,30,30,32,41,51,61,65,67$, $70,72,73,74,77,79,80,81,87,87,88,89,91,93,96,97,100,101,104$, $104,108,109,120,131,150,157,167,231,240,400$.

Data set 2:

The data set 2 in Table 2 represents the life time's data relating to times (in months from $1^{\text {st }}$ January, 2013 to $31^{\text {st }}$ 
July, 2018) of 105 patients who were diagnosed with hypertension and received at least one treatment related to hypertension in the hospital where death is the event of interest. The data has been used by Umeh and Ibenegbu [14]. The data set is as follows:

Table 2. The table showing the data set of patients who were diagnosed with hypertension.

$45,37,14,64,67,58,67,55,64,62,9,65,65,43,13,8,31,30,66,9,10$, $31,31,31,46,37,46,44,45,30,26,28,45,40,47,53,47,41,39,33,38$, $26,22,31,46,47,66,61,54,28,9,63,56,9,49,52,58,49,53,63,16,67$, $61,67,28,17,31,46,52,50,30,33,13,63,54,63,56,32,33,37,7,56,1$, $67,38,33,22,25,30,34,53,53,41,45,59,59,60,62,14,57,56,57,40$, 44,63

Table 3. The MLEs and goodness of fit statistics for all fitted models for the data set 1.

\begin{tabular}{llllll}
\hline Models & MLE & LL & AIC & AICC & BIC \\
\hline \multirow{2}{*}{ TLExIEx } & $\begin{array}{l}\alpha=4.8084 \\
\theta=0.2337\end{array}$ & -240.4835 & 486.9669 & 487.4776 & 492.7624 \\
& $\begin{array}{l}\beta=7.7843 \\
\theta=0.5713\end{array}$ & & & & \\
TLIEx & $\begin{array}{l}\theta=37.4297 \\
\text { ExIEx }\end{array}$ & -323.8932 & 651.7863 & 652.0363 & 655.6500 \\
IEx & $\alpha=4.1563$ & & & & \\
Ex & $\beta=17.1813$ & -306.1066 & 616.2133 & 616.4633 & 620.0769 \\
\hline
\end{tabular}

Table 4. The MLEs and goodness of fit statistics for all fitted models for the data set 2.

\begin{tabular}{|c|c|c|c|c|c|}
\hline Models & MLE & $\mathbf{L L}$ & AIC & CAIC & BIC \\
\hline TLExIEx & $\begin{array}{l}\alpha=7.0862 \\
\theta=0.3235 \\
\beta=5.2821\end{array}$ & -417.6828 & 841.3655 & 841.6031 & 849.3274 \\
\hline TLIEx & $\begin{array}{l}\theta=0.9352 \\
\beta=37.4299\end{array}$ & -518.4823 & 1040.9650 & 1041.0820 & 1046.2720 \\
\hline ExIEx & $\begin{array}{l}\beta=4.8724 \\
\alpha=4.9481\end{array}$ & -525.8110 & 1055.6220 & 1055.7400 & 1060.9300 \\
\hline IEx & $\beta=24.1153$ & -525.8110 & 1053.6220 & 1053.6610 & 1056.2760 \\
\hline Ex & $\beta=0.0237$ & -497.8593 & 997.7186 & 997.7576 & 1000.3730 \\
\hline
\end{tabular}

\section{Conclusion}

In this article, we derived and studied a new probability distribution called the Topp Leone Exponentiated Inverse Exponential distribution using the family of distributions proposed by Ibrahim et al., [12]. Some of its mathematical and statistical properties comprising the moments, moment generating function, quantile function, order statistic and reliability analysis were derived. The parameters of the distribution were also obtained using method of maximum likelihood estimate. We used two real-life data sets to test the fitness of the new distribution with some other known distributions comprising of exponential, inverse exponential, exponentiated inverse exponential, Topp Leone inverse exponential and Topp Leone exponentiated inverse exponential distributions. The results showed that the new distribution which is the Topp Leone exponentiated inverse exponential distribution is the best distribution for fitting the two data sets among the distributions considered in this article.

\section{References}

[1] G. S. Mudholkar and D. K. Srivastava, 1993, Exponentiated Weibull family for analyzing bathtub failure data, IEEE Transactions on Reliability, 42, 299-302.

[2] R. C. Gupta, P. L. Gupta and R. D. Gupta, 1998, Modeling failure time data by Lehmann alternatives. Communications in Statistics-Theory and Methods, 27, 887-904.

[3] R. C. Gupta and D. Kundu, 2001, Exponentiated exponential family; an alternative to gamma and Weibull, Biometrical Journal, 43, 117-130.

[4] A. S. Hassan and M. Abd-Allah, 2018, Exponentiated Weibull-Lomax Distribution: Properties and Estimation. Journal of Data Science, 277-298.

[5] R. Jan, T. R. Jan and P. B. Ahmad, 2018, Exponentiated Inverse Power Lindley Distribution and its Applications. arXivpreprintarXiv: 1808.07410.

[6] A. S. Hassan and M. A. Abdelghafar, 2017, Exponentiated Lomax geometric distribution: Properties and applications. Pakistan Journal of Statistics and Operation Research, 13, 3, 545-566.

[7] A. H. El-Bassiouny, M. El-Damcese, A. Mustafa and M. S. Eliwa, 2017, Exponentiated generalized Weibull-Gompertz distribution with application in survival analysis. Journal of Statistical Application and Probability, 6, 7-16.

[8] A. Z. Keller and A. R. Kamath, 1982, Reliability analysis of CNC Machine Tools. Reliability Engineering, 3, 449-473.

[9] P. E. Oguntunde and A. O. Adejumo, 2015, The transmuted inverse exponential distribution. International Journal of Advanced Statistics and Probability, 3, 1, 1-7. doi: 10.14419/ijasp.v3i1.3684.

[10] P. E. Oguntunde, A. O. Adejumo and E. A Owoloko, 2017, Application of Kumaraswamy inverse exponential distribution to real-life time data. International Journal of Applied Mathematics and Statistics, 56, 5, 34-47.

[11] P. E. Oguntunde, A. O. Adejumo and E. A. Owoloko, 2017, July 5-7, On the flexibility of the transmuted inverse exponential distribution. Lecture Notes on engineering and computer science: Proceeding of the World Congresson Engineering (123-126). London, UK.

[12] S. Ibrahim, S. I. Doguwa, I. Audu and H. M. Jibril, 2020, On the Topp Leone exponentiated-G Family of Distributions: Properties and Applications. Asian Journal of Probability and Statistics, 7, 1, 1-15.

[13] J. P. Klein and M. L. Moeschberger, 2003, Survival analysis: technique for censored and truncated data $\left(2^{\text {nd }}\right.$ Edition $)$, Springer, New York, USA, 535 Pages, ISBN978-0-38721645-4.

[14] E. Umeh and A. Ibenegbu, 2019, A Two-Parameter Pranav Distribution with Properties and Its Application; Journal of Biostatistics and Epidemiology, 5, 1, 74-90. 